\title{
The middle Pleistocene transition by frequency locking and slow ramping of internal period
}

\author{
Karl H. M. Nyman ${ }^{1}$ (D) Peter D. Ditlevsen ${ }^{1}$
}

Received: 24 August 2018 / Accepted: 9 February 2019 / Published online: 22 March 2019

(c) The Author(s) 2019

\begin{abstract}
The increase in glacial cycle length from approximately 41 to on average 100 thousand years around 1 million years ago, called the middle Pleistocene transition (MPT), lacks a conclusive explanation. We describe a dynamical mechanism which we call ramping with frequency locking (RFL), that explains the transition by an interaction between the internal period of a self-sustained oscillator and forcing that contains periodic components. This mechanism naturally explains the abrupt increase in cycle length from approximately 40 to 80 thousand years observed in proxy data, unlike some previously proposed mechanisms for the MPT. A rapid increase in durations can be produced by a rapid change in an external parameter, but this assumes rather than explains the abruptness. In contrast, models relying on frequency locking can produce a rapid change in durations assuming only a slow change in an external parameter. We propose a scheme for detecting RFL in complex, computationally expensive models, and motivate the search for climate variables that can gradually increase the internal period of the glacial cycles.
\end{abstract}

Keywords Glacial cycles · Middle Pleistocene transition · Frequency locking · Internal period · Abrupt transition

\section{Introduction}

Since the beginning of major Northern hemisphere glaciation 2.7 million years (Myr) ago, Earth has undergone alternating epochs of icy and cold conditions on the one hand, and warm and ice-free conditions on the other (Fig. 1). While these glacial cycles are attested from geological records (EPICA Community Members 2004; Huybers 2007; Lisiecki and Raymo 2005), there is no single conclusive theory of their origins.

Milutin Milanković proposed in the 1920s that glacial cycles should repeat roughly every 40 thousand years (kyr), based on calculations of incoming solar radiation. Alas, in the 1970s accurately dated oxygen isotope data from ocean sediment cores cast doubt on his theory, by revealing that the glacial cycles over the past $800 \mathrm{kyr}$ were closer to 100 kyr long (Imbrie and Imbrie 1979). Soon it became clear,

Karl H. M. Nyman

karl.nyman@nbi.ku.dk

Peter D. Ditlevsen

pditlev@nbi.ku.dk

1 Centre for Ice and Climate, Niels Bohr Institute, University of Copenhagen, Copenhagen, Denmark however, that the $\sim 100 \mathrm{kyr}$ cycles were preceded by $\sim 40$ kyr long cycles, consistent with the theory of Milanković. This spawned three questions: what caused the $\sim 100 \mathrm{kyr}$ cycles, what caused the $\sim 40$ kyr cycles (was Milanković right?), and what caused the transition between them around 1 Myr ago, called the middle Pleistocene transition (MPT) (Clark et al. 2006). The last question is the focus of this paper.

The main strategy to address these questions has been to replicate the palaeoclimatic records using simple models of glacial cycles with few variables, referred to as conceptual models [see (Crucifix 2012) for a review]. One reason for this is that the rather regular and cyclic variations in data suggest that the main dynamics can be captured by a system of few degrees of freedom, even as the full climate system obviously has a large number of degrees of freedom. None of these models describe the climate system in detail, but they are useful for understanding underlying dynamics. Virtually all models involve insolation variations due to changes to Earth's orbital configuration relative to the sun, an idea heralded by Adhémar, Croll and Milankovitch (Imbrie and Imbrie 1979). But the specific role played by insolation variations is still unknown and debated. 


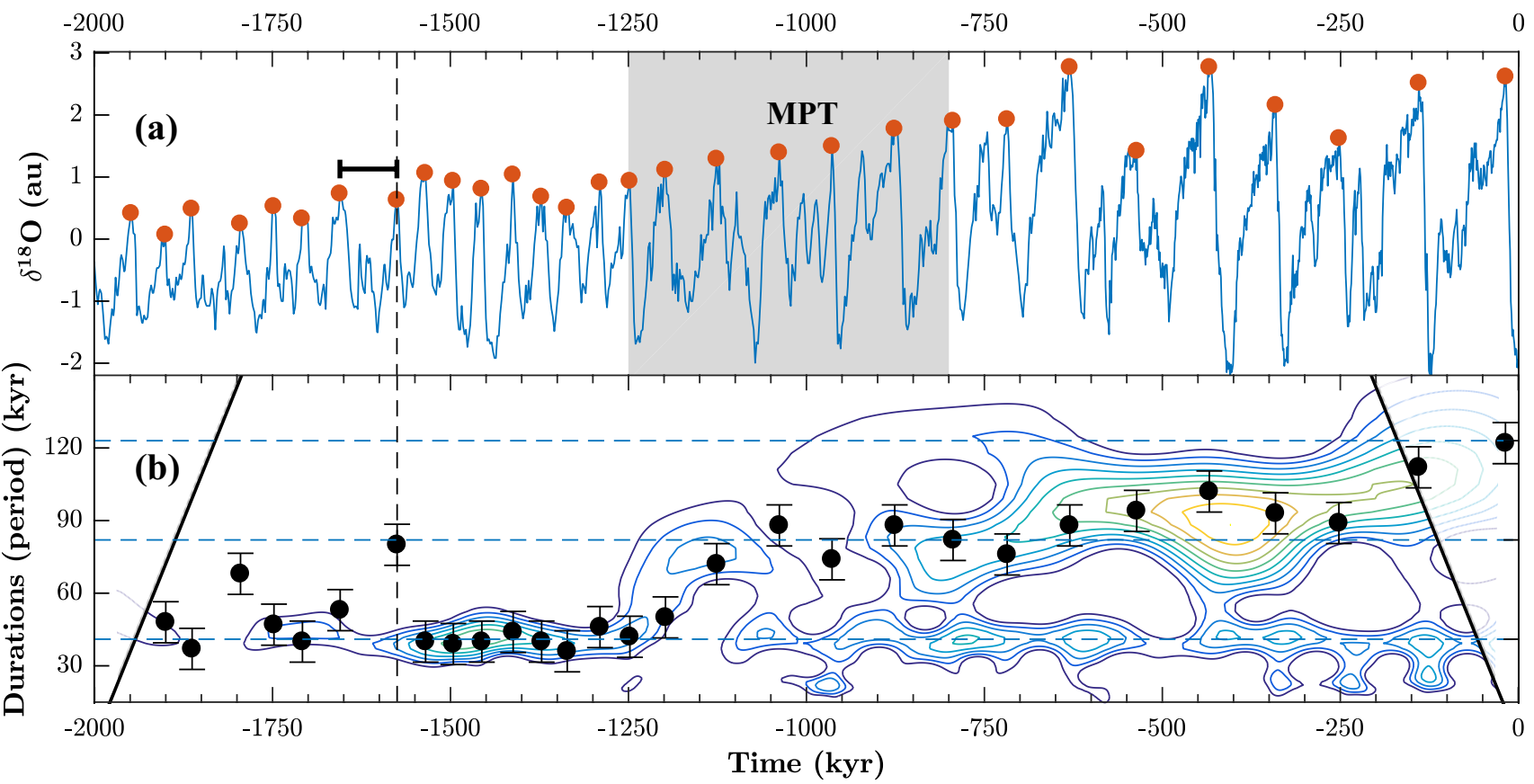

Fig. 1 a The LR04 stack of normalised isotopic oxygen anomalies in deep ocean sediment cores-a proxy for global ice volume and deep ocean temperatures (Lisiecki and Raymo 2005). Higher values means more ice. b Durations between successive major terminations (black), showing an increase at the MPT. Error bars indicate one standard deviation dating uncertainty. Contours (darker to lighter) show the amplitude of a wavelet spectral estimate. Each lighter contour corresponds to an increase of $7 \%$ of the maximum amplitude, starting at $30 \%$. Outside the cone of influence (thick black line), edge effects are important. See "Appendix B and D" for details
Several solutions to the cause of the MPT have been presented within the context of conceptual models. Some mechanisms rely on a bifurcation occurring in the unforced climate system which fundamentally changes how the system operates (Ashwin and Ditlevsen 2015; Ditlevsen 2009; Huybers and Langmuir 2017; Maasch and Salzman 1990; Tziperman and Gildor 2003). Other mechanisms invoke a "spontaneous" change, such as a shift between attractors due to subtle changes in insolation (Omta et al. 2015; Quinn et al. 2018) or random fluctuations (Imbrie et al. 2011; Salzman and Verbitsky 1993), or as a coincidence (Huybers 2009). A third possible mechanism for the MPT assumes one essential mode of oscillation throughout the Pleistocene and relies crucially on the interaction between insolation variations and an increasing internal period. This mechanism, previously imprecisely referred to as phase/frequency locking and non-linear resonance-but here ramping with frequency locking (RFL) - is the focus of this paper.

The main appeal of this mechanism is that nothing special had to occur in the climate system over the MPT (Huybers 2007); it is only required that the internal period was ramped slowly-interactions with forcing are enough to cause an abrupt increase in durations between glacial terminations (Fig. 1, bottom panel).

The first publications where RFL was used (Paillard 1998; Paillard and Parrenin 2004) did not explain why the durations between glacial transitions increased abruptly over the MPT. Ashkenazy (2006) and Ashkenazy and Tziperman (2004) hinted how frequency locking (therein called phase locking) could produce an abrupt increase in duration, by showing diagrams of average duration as a function of a system parameter (Devil's staircases). Huybers (2007) was first to both show a model trajectory of ice volume using the mechanism, and to attribute the effect to "skipping of obliquity cycles", a frequency locking effect. Recently, Daruka and Ditlevsen (2015), Feng and Bailer-Jones (2015), Mitsui et al. (2015) and Tzedakis et al. (2017) alluded to the mechanism, but neither emphasised that frequency locking can explain the MPT assuming only a slow linear change in a climate parameter. Instead, by ramping some parameter in a way that mimics the rapid change in durations over the MPT, they prescribe an abrupt increase in period over the MPT rather than explaining it. Here, we for the first time properly define RFL and emphasise its generality.

Following (Huybers 2007), we question the common assumption that climate entered a stationary state in the late Pleistocene, and instead argue that the sequence of durations between glacial terminations is consistent with a slow increase of the internal period of the climate system until present (Fig. 1b). According to this view, the typical durations between transitions changed from $\sim 40$ to $\sim 80 \mathrm{kyr}$ around $1200 \mathrm{kyr}$ ago, after which they increased gradually 
in the mean to present time, with the last duration being $\sim 120$ kyr long.

Here, we first aim to explain RFL in a clear way, using a harmonically forced simple model. We use harmonic (pure sine) forcing because it makes frequency locking concepts clearer, while still producing qualitatively similar behaviour to astronomical forcing curves. We should not expect model runs with such simplified forcing to agree well with data, however. We use forcing with period $41 \mathrm{kyr}$, corresponding to the main period of obliquity variations (Berger 1978), which determine the total insolation integrated over the summer at Northern latitudes (Huybers 2006).

We then define RFL, specify a class of models able to reproduce the MPT using the mechanism, and propose a decomposition of model components to understand the abruptness of the MPT. We consider evidence in data for a 40-80 kyr shift in durations between terminations and a subsequent gradual increase, and why this supports RFL in favour of some other mechanisms for the MPT. We then discuss how insights from harmonic forcing relate to nonharmonic forcing, how RFL can be detected in complex and computationally expensive models, and some climate variables that can cause an increase in the internal period of the glacial cycles.

\section{The idea behind ramping with frequency locking}

We illustrate RFL using a deterministic and continuous time version of the H07 model (Huybers 2007) (see Fig. 2). The model is arguably the simplest to represent alternating stages of intrinsic growth and decay of ice sheets, with the growth state ending abruptly as a critical ice volume is reached. It is is an integrate-and-fire threshold model conceptually very similar to the models in Ashkenazy and Tziperman (2004), Glass and Mackey (1979), Huybers (2007), Imbrie and Imbrie (1980), Imbrie et al. (2011), Paillard (1998),
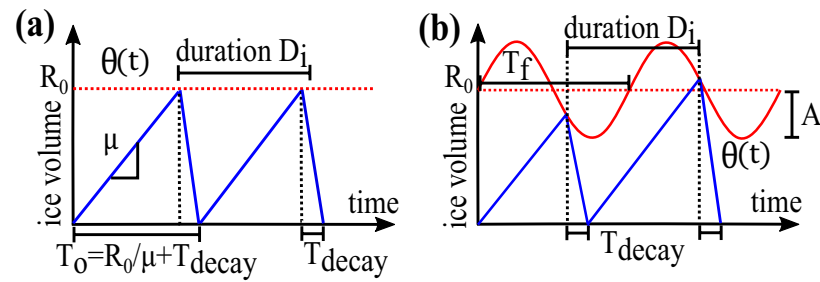

Fig. 2 The H07 model (Eq. 1), a unforced and b periodically forced. Ice volume (blue) grows linearly at a rate $\mu$ until a threshold (red) is hit, after which ice volume is reset to 0 over a time $T_{\text {decay }}$. In a the threshold of glacial termination $\theta(t)$ is constant $\theta(t)=R_{0}$, whereas in $\mathbf{b}$ it oscillates periodically as $\theta(t)=R_{0}+A \sin \left(2 \pi t / T_{f}\right)$. $T_{o}$ is the internal (unforced) period of the model
Parrenin and Paillard (2003, 2012), van der Pol and van der Mark (1927) and de Saedeleer et al. (2013).

Physically, sudden and rapid deglaciation has been explained e.g. with isostatic rebound, rapid $\mathrm{CO}_{2}$ outgassing (Paillard and Parrenin 2004) and rapid loss of Northern hemisphere sea ice cover (Gildor and Tziperman 2000).

We assume that ice volume $x(t)$ grows at a constant rate $\mu$ in a glacial state until it reaches a threshold $\theta(t)$. Then deglaciation starts, whereby ice volume decays to 0 over a fixed time $T_{\text {decay }}=10 \mathrm{kyr}$ :

$\dot{x}=\mu$ until $x(t)=\theta(t)$, then

linearly decrease $x(t)$ to 0 over time $T_{\text {decay }}$, repeat.

Small perturbations to the model, such as having a constant rate of decay instead of a fixed time, does not qualitatively affect its behaviour.

We split $\theta(t)$ into a forcing term $A \cdot F(t)$-a zero-mean sum of periodic components-and a ramping term $R(t)$ : $\theta(t)=R(t)+A \cdot F(t)$

In the limit of constant ramping $R(t)=R_{0}$ and zero forcing $A=0$, the system has a constant internal period of oscillation $T_{o}=\frac{R_{0}}{\mu}+T_{\text {decay }}$ (subscript $o$ for oscillator), see Fig. 2a. But if the threshold increases slowly over time, for instance linearly $\theta(t)=R(t)=R_{0}+R_{1} t$ as in Fig. 3ai, then the internal period $T_{o}(t)=\frac{R_{0}}{\mu}+T_{\text {decay }}+\frac{R_{1}}{\mu} t$ also increases slowly (Fig. 3bi). As there is no forcing, the durations between glacial terminations follow $T_{o}(t)$ closely.

However, with periodic forcing

$\theta(t)=R_{0}+R_{1} t+A \sin \left(2 \pi / T_{f}\right)$, durations $D_{i}$ are near multiples of the forcing period $D_{i} \approx N T_{f}, N \in \mathbb{N}$ (Fig. 3bi). Roughly speaking, the multiple that is realised is the one closest to the internal period $T_{o}$. This phenomenon, called frequency locking (Pikovsky et al. 2001), has been studied extensively over the past century [e.g. Cartwright and Littlewood (1945); Glass and Mackey (1979); Le Treut and Ghil (1983); van der Pol and van der Mark (1927); Tziperman et al. (2006)].

In Fig. 3ai the durations $D_{i}$ change abruptly from $1 \times T_{f}$ to $2 \times T_{f}$ and finally $3 \times T_{f}$. These abrupt changes in durations resulting from a gradual change in an underlying parameter is one possible dynamical mechanism behind the MPT.

We call the mechanism RFL, rather than non-linear resonance, phase locking or frequency locking as it has previously been called. This we do to emphasise both that an internal period must increase gradually over time (ramping), and that the internal oscillations must be locked to external forcing. This is opposed to e.g. the mechanism in Omta et al. (2015), which realises the MPT through jumps between coexisting frequency locked solutions.

We note that RFL is a special case of "slow passage through bifurcation" [e.g. (Baer et al. 1989; Do and Lopez 2012)], for 


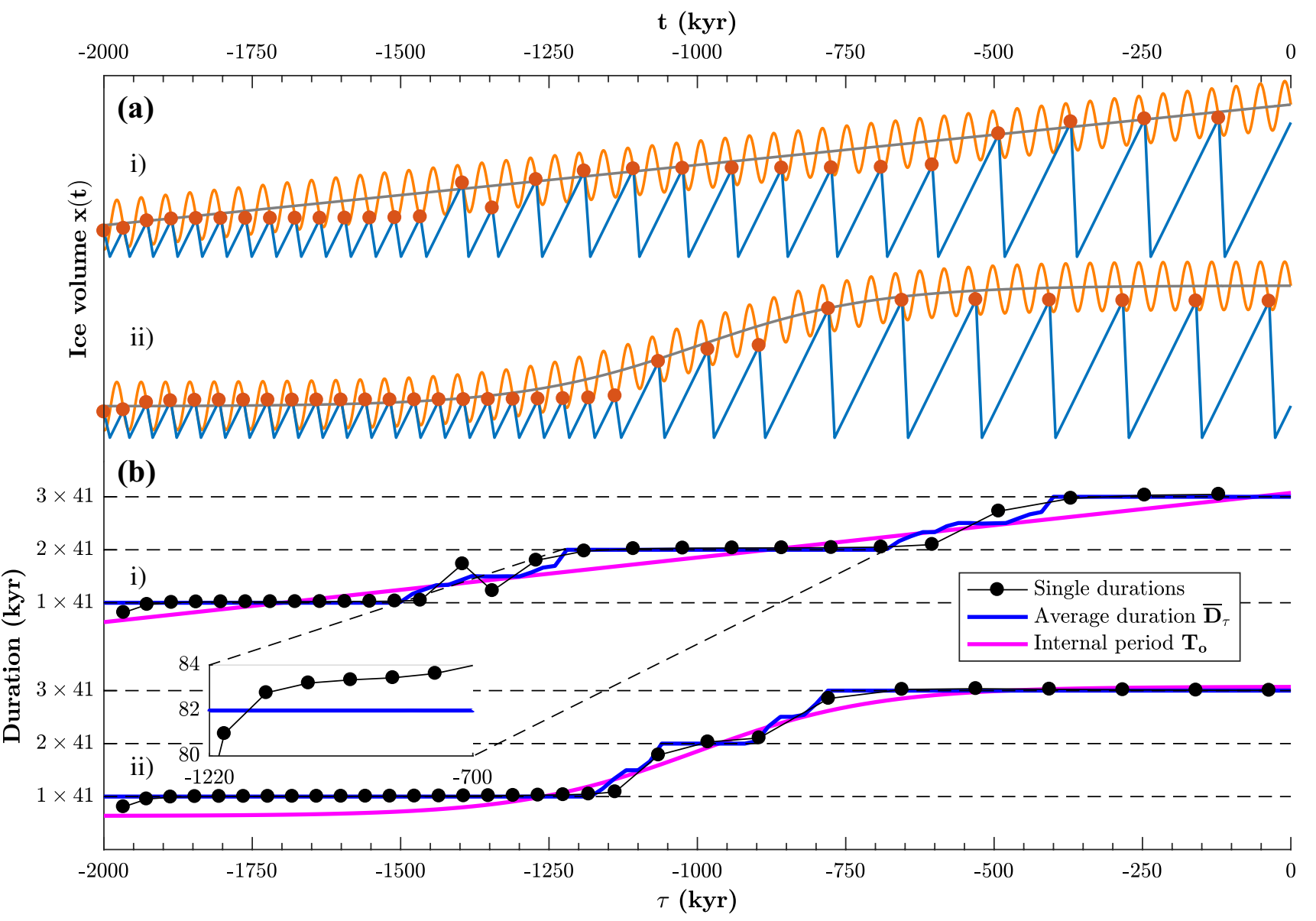

Fig. 3 The ramping with frequency locking (RFL) mechanism for the periodically forced $\mathrm{H} 07$ model. a Ice volume (blue sawtooth) over time for a i) linear and ii) sigmoidal ramp of the upper threshold $\theta(t)$. Periodic threshold in orange and unforced threshold in grey. b Average duration between glacial terminations $\bar{D}_{\tau}$ over frozen time $\tau$ (black solid) (known as Devil's staircases, Sect. 4.1) for the i) linearly and ii) sigmoidally ramped thresh-

which the bifurcations typically are saddle-node bifurcations of limit cycles marking transitions in and out of frequency locking regions (Pikovsky et al. 2001). [However, see e.g. Guckenheimer et al. (2003) and Levi (1990) for other relevant bifurcations].

Finally, we note that $\mathrm{H} 07$ is an illustrative example of RFL, and not representative of all glacial cycle models. However, the rapid jumps between frequency locking regions occur generically in a broad class of models, defined next.

\section{A formal description of RFL}

H07 (Fig. 2) is just one particular model capable of realising the MPT through RFL. We could simply call these selfsustained oscillators, but we aim to be more precise and to establish notation. olds, and sample durations (red dotted lines) for the forced solutions in a). Magenta lines show the internal period $T_{o}(\tau)$. The inset shows that durations with time-varying ramping $R(t)$ do not agree perfectly with the average duration $\bar{D}_{\tau}$, computed for $R(t)=\mathrm{const}$ (see Sect. 5). Parameters are $\mu=1, T_{\text {decay }}=10, T_{f}=41, A=20$ and ramp functions are i) $R(\tau)=26+0.05 \times(\tau+2000)$ and ii) $R(t)=26+50\left(\tanh \left(\frac{t+1000}{300}\right)+1\right)$ respectively

First, we naturally require the model to be a dynamical system, such that there is an evolution rule $f(t, x)$ taking a state $x(t)$ forward in time $t$. We identify the model with the evolution rule and denote it $f$ (without arguments) for brevity. $f(t, x), x(t)$ and $t$ can be very general, for instance; $t$ can be continuous or discrete, $x$ can be of any dimension, and $f(t, x)$ can e.g. be a piecewise smooth ODE paired with a switching rule, as for $\mathrm{H} 07$.

We also require $f$ to be forced by a continuous zeromean sum of periodic components $A(t) F(t)$ with an amplitude $A(t)$, called the forcing. We further require that $f$ is parametrised by a set of parameters $p(t)$, whose time-varying subset $R(t)$ is called the ramping. Thus we can write $f=f(t, x, R(t), A(t) F(t))$.

We define the frozen system $f_{\tau}:=f(t, x, R(\tau), A(\tau) F(t))$ as $f$ with parameters frozen at time $t=\tau$. Importantly, we require that $f$ is a self-sustained oscillator with internal period 
$T_{o}(R(\tau))$, meaning that every solution to $f_{\tau}$ with $A(t) \equiv 0$ tends asymptotically (as $t \rightarrow \infty$ ) to a periodic solution with period $T_{o}(R(\tau))$. For RFL to be relevant we require that $T_{o}(R(\tau))$ increases as a function of $\tau$. This is the ramping part of RFL.

The frequency locking part of RFL comes from the response of $f$ to non-zero but constant forcing $A(\tau)$. For small and medium size $A(\tau)$, asymptotic solutions to the frozen system $f(t, x, R(\tau), A(\tau) F(t))$, are generically periodic with periods related rationally to the forcing periods (Pikovsky et al. 2001). The oscillator period can for instance be twice that of the forcing period. If so, the oscillator period (and therefore frequency) remains constant on open sets of parameters and we say that solutions are frequency locked to the forcing (we return to this in Sect. 4).

The essence of RFL is that the period of the frozen system can change rapidly as function of $T_{o}(R)$ when a ramped parameter causes the system to switch between frequency locking regions.

However, some remarks are in place. Firstly, the system with time varying parameters $f(t, x(t), R(t), A(t) F(t))$ is not the same as the frozen system $f(t, x(t), R(t), A(\tau) F(\tau))$ since solutions to the former cannot equilibrate to solutions of the latter in finite time. We return to differences between the two systems in Sect. 5 but until then we focus on the frozen system.

Secondly, the period of an oscillator is not the same as the length of individual "cycles". For instance, around - 1350 kyr in Fig. 3, short and long "cycles" alternate. This makes the average time between terminations $61.5 \mathrm{kyr}$, whereas the period (time until repetition, two large peaks) is $123 \mathrm{kyr}$. Therefore, we instead characterise local behaviour with the average duration:

$\bar{D}_{\tau}=\lim _{n \rightarrow \infty} \frac{1}{n} \sum_{i=1}^{n} D_{i, \tau}$

where $D_{i, \tau}$ denotes the $i$ :th duration between successive crossings of a fixed threshold for the frozen system $f_{\tau}$, and the limit is taken as the number of crossings $n$ goes to infinity. The threshold should be chosen such that a crossing occurs once per glacial cycle. For some models, like H07, the threshold defining glacial terminations can be used as a threshold to define durations $D_{i, \tau}$. For models without explicit thresholds, an appropriately chosen Poincaré section can be used instead (Pikovsky et al. 2001).

\section{Breaking down the dependency of $\bar{D}_{\tau}$ on $\tau$}

Comparing Fig. 3bi and ii shows that $\bar{D}_{\tau}$ can rise steeply both from frequency locking effects under a gradual change of parameter (Fig. 3bi) and from ramping of a climate parameter rapidly (Fig. 3bii).
We wish to break down the contribution to the local change in average duration from these effects and do so by considering the change $\Delta \bar{D}_{\tau}$ under a small perturbation $\Delta \tau$ :

$$
\begin{aligned}
\frac{\Delta \bar{D}_{\tau}(\tau)}{\Delta \tau} \approx & \frac{\bar{D}_{\tau}(\tau+\Delta \tau)-\bar{D}_{\tau}(\tau)}{\Delta \tau} \\
\approx & \frac{\Delta \bar{D}_{\tau}\left(T_{o}, A\right)}{\Delta T_{o}} \frac{\Delta T_{o}(R)}{\Delta R} \frac{\Delta R(\tau)}{\Delta \tau} \\
& +\frac{\Delta \bar{D}_{\tau}\left(T_{o}, A\right)}{\Delta A} \frac{\Delta A(\tau)}{\Delta \tau},
\end{aligned}
$$

where e.g. $\frac{\Delta \bar{D}_{\tau}\left(T_{o}, A\right)}{\Delta T_{o}}:=\frac{\bar{D}_{\tau}\left(T_{o}+\Delta T_{o}, A\right)-\bar{D}_{\tau}\left(T_{o}, A\right)}{\Delta T_{o}}$, and where we have neglected higher order terms. This approximation is generally better the smaller $\Delta \tau$ is. As $\Delta \tau \rightarrow 0$, (3) tends to the chain rule, but since $\frac{\Delta \bar{D}_{\tau}\left(T_{o}, A\right)}{\Delta T_{o}}=0$ wherever differentiable (see Sect. 4.1) it is more appropriate to consider $\Delta \bar{D}_{\tau}$ over short intervals of time $\Delta \tau$. In what follows we restrict ourselves to $\frac{\Delta A(\tau)}{\Delta \tau}=0$.

Equation (3) says that the rate of change (abruptness) in time of the average duration $\bar{D}_{\tau}$ is approximately the product of the rates at which $R(t)$ changes with time, $T_{o}$ changes with $R$, and $\bar{D}_{\tau}$ changes with $T_{o}$. Our point is that each of these factors can contribute to an abrupt change of $\bar{D}_{\tau}$ at the MPT, but they have different interpretations from a modelling perspective. We discuss these factors next.

\section{1 $\bar{D}_{\tau}\left(T_{o}, A\right)$ : Arnold tongues and Devil's staircases}

The average duration $\bar{D}_{\tau}\left(T_{o}, A\right)$ as a function of internal period $T_{o}$ and forcing amplitude $A$ describes the frequency locking contribution to changes to $\bar{D}_{\tau}$ over time $\tau$.

Frequency locking can be visualised in Arnold tongue diagrams; Fig. 4a reveals regions of constant average duration $\bar{D}_{\tau}$ in $\left(T_{o}, A\right)$ space called Arnold tongues (Crucifix 2013; Pikovsky et al. 2001; de Saedeleer et al. 2013). Inside major $1: N$ tongues, solutions are periodic with period $N$ times the forcing period $T_{f}=41 \mathrm{kyr}$, as evidenced in Fig. 4a. Minor tongues emanate at $A=0$ from other rationals of $T_{f}$, and in between them are quasiperiodic solutions. (We show only $M: N, M=\{1,2\}$ Arnold tongues, defined numerically as sets for which $\left|\bar{D}_{\tau}-T_{f} \frac{N}{M}\right|<0.5$. $\bar{D}_{\tau}$ is estimated over 6 Myr.)

A change in $\tau$ that in turn leads to a change in $T_{o}(R(\tau))$ traces out a path in $\left(A, T_{o}\right)$ space (black and magenta lines in Fig. 4a. Such a path represents the change in system state as one or more parameters change in time over the MPT. The paths in Fig. 4a pass through the major 1:1, 1:2 and 1:3 locking tongues, in which there are respectively 1,2 and 3 forcing periods per oscillator period. We learn that for larger $A$, a larger portion of the path stays inside the major $1: N$ tongues, an observation also made in (Ashkenazy 2006). 

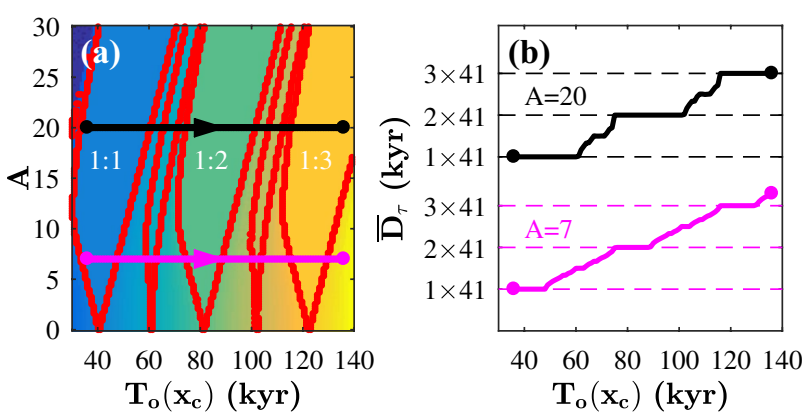

Fig. 4 a Arnold tongue diagram for increasing upper threshold $R_{0}$ and periodic forcing $\left(T_{f}=41 \mathrm{kyr}\right)$ in the $\mathrm{H} 07$ model, showing regions in $\left(A, T_{o}\right)$ space of constant average duration $\bar{D}_{\tau}$ (enclosed by red dots). Major 1:N tongues, meaning that $\bar{D}_{\tau}=N T_{f}$, are labelled. Colour scale from blue (short) to yellow (long) reflects average duration. b Comparison between average duration $\bar{D}_{\tau}$ as a function of internal period $T_{o}$ for strong $(A=20)$ and weaker $(A=7)$ forcing

Another way of visualising the change in average duration $\bar{D}_{\tau}$ as a function of $T_{o}$ are Devil's staircases (Pikovsky et al. 2001) (Fig. 4b), in which the forcing amplitude $A$ is fixed. We see that the average duration $\bar{D}_{\tau}$ is constant within Arnold tongues and that the staircase for larger $A$ contains longer steps of constant duration, as predicted from Fig. 4a. Hence, stronger forcing influence tends to cause more abrupt changes to the average period.

\section{$4.2 T_{o}(R)$ and $R(\tau)$}

The function $T_{o}(R)$, if continuous and monotonic, stretches and squeezes Arnold tongues by scaling the independent variable $T_{o}$ of $\bar{D}_{\tau}\left(T_{o}\right)$. In the Ashkenazy model (Ashkenazy 2006), for instance, a faster-than-linearly increasing $T_{o}(R)$ makes the 1:2 and 1:3 Arnold tongues, as a function of ice volume threshold, narrower and more closely spaced than the 1:1 tongue.

Ramping $R(\tau)$ continuously and monotonically, similarly stretches and squeezes Arnold tongues. For instance, in Fig. 5a sigmoidal ramping $R(t)$ makes the 1:2 Arnold tongue narrower compared to a linear change of $R(t)$. Figure 3 further illustrates this, showing model runs for either a sigmoidally $(R(t)=26+50 \times(\tanh ((t+1000) / 300)+1))$ or a linearly $R(t)=26+0.05 \times(t+2000)$ ramped threshold. The sigmoidal ramping accelerates the increase in average duration around $-1000 \mathrm{kyr}$, making the transition more abrupt. Note that the parameters in the functions $R(\tau)$ in Figs. 3 and 5 are different.

\subsection{The roles of $\bar{D}_{\tau}\left(T_{o}, A\right), T_{o}(R)$ and $R(\tau)$ in reproducing the MPT}

All of $\bar{D}_{\tau}\left(T_{o}, A\right), T_{o}(R)$ and $R(\tau)$ govern the average duration $\bar{D}_{\tau}$ and are able to cause an abrupt change of it, like the one observed at the MPT. From a modelling point of view,
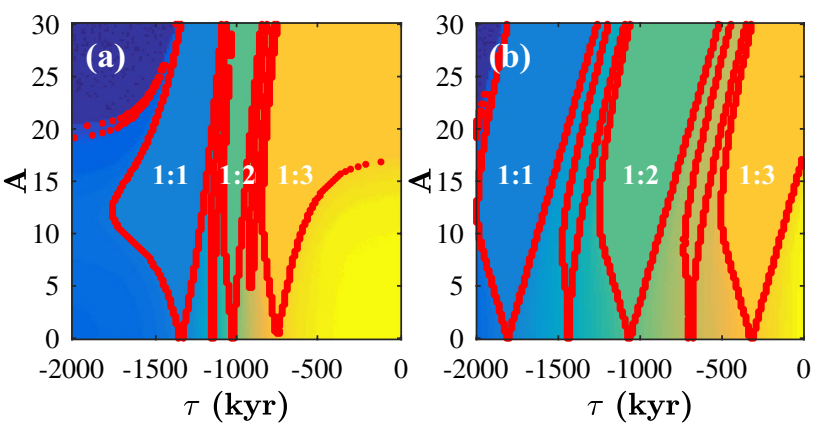

Fig. 5 Stretching of Arnold tongues by ramping the threshold parameter $R(\tau)$ in $\mathrm{H} 07$ at different rates. In a $R(\tau)$ is ramped sigmoidally $R(\tau)=20+55 \times\left(\tanh \left(\frac{\tau+1000}{300}\right)+1\right)$, while in b $R(\tau)=20+$ $0.055 \times(\tau+2000)$. For further details, see Fig. 4 and the text

however, the functions carry different assumptions and are compatible with different hypotheses.

A model having an abrupt change due to $\bar{D}_{\tau}\left(T_{o}, A\right)$ relies on frequency locking properties, and assumes only slowly varying functions $T_{o}(R)$ and $R(\tau)$. Hence, the internal period is assumed to change slowly with model parameters, and parameters are assumed to change slowly in time. Such a model, relying on few assumptions about the climate system, makes full use of the RFL mechanism. The models in Huybers (2007), Paillard (1998) and Paillard and Parrenin (2004) and H07 are of this kind.

A model which relies predominantly on $T_{o}(R)$ for an abrupt change in average duration $\bar{D}_{\tau}$ is also consistent with a slowly changing external parameter $R(\tau)$, but a particular function $T_{o}(R)$ requires a physical explanation.

A model relying on a rapidly changed external parameter $R(\tau)$ does not need frequency locking properties of $\bar{D}_{\tau}\left(T_{o}, A\right)$ or a non-linear response of internal dynamics to the parameter $T_{o}(R)$. However, such a model prescribes the abrupt change in average duration at the MPT rather than explaining the dynamics behind it. Therefore, such an explanation requires justification for the rapidly changed external parameter. The models in Ashkenazy and Tziperman (2004), Daruka and Ditlevsen (2015), Mitsui et al. (2015) and Tzedakis et al. (2017) can be said to fall under this category, although they also achieve some abruptness through $\bar{D}_{\tau}\left(T_{o}, A\right)$.

\section{Validity of the quasi-static approximation $\boldsymbol{f} \sim \boldsymbol{f}_{\tau}$}

The quasistatic approximation is the approximation that parameters $R(\tau)$ change so slowly that the local average duration of $f$ at time $t=\tau$ : 
$\bar{D}_{\tau, l o c}=\frac{1}{|I(\tau)|} \sum_{i \in I(\tau)} D_{i, \tau}$,

is equal to $\bar{D}_{\tau} . I(\tau)$ is the set of indices of durations $D_{i, \tau}$ within a time interval $\left[\tau-\tau_{0}, \tau+\tau_{1}\right]$ around $\tau$, with $\tau_{0}, \tau_{1}>0$. If $I(\tau)=\emptyset$, then we define $\bar{D}_{\tau, l o c}=0$.

If the quasistatic approximation holds, then the average duration, Arnold tongue diagrams and Devil's staircases calculated for the frozen system $f_{\tau}$ provide accurate information about local dynamics of $f$.

However, if $R(t)$ and/or $T_{o}(R(t))$ change rapidly around $t=\tau$, then there are two sources of discrepancy between $\bar{D}_{\tau, l o c}$ and $\bar{D}_{\tau}$.

The first comes from that the length of the interval of time needed for a good average may be long relative to the local change of $\bar{D}_{\tau}$ for the system $f_{\tau}$. Figure 6 illustrates that for a $9 \times 41=369$ kyr-periodic solution, a long interval is needed to get a local average duration $\bar{D}_{\tau, l o c}$ in agreement with $\bar{D}_{\tau}$. At the same time, a long averaging interval fails to capture abrupt changes to $\bar{D}_{\tau}$.

The second is that solutions to $f$ may fail to track solutions to $f_{\tau}$. This occurs if the "frozen" attractor of $f_{\tau}$ changes (in some sense) at a fast rate, and if solutions attract to the frozen attractor at a slow rate. Quantifying these rates in a coordinate- and model-independent way seems difficult, however.

A candidate measure of rate of attraction is the maximal Lyapunov exponent of the return map mapping one transition time to another (Pikovsky et al. 2001). This can be normalised to a common time scale between models and is coordinate independent. However, since it is only a local measure it neglects the time it takes to enter a small neighbourhood of the attractor. This time can in practice dominate, as is the case in the standard circle model (not shown, model described in Pikovsky et al. 2001).

The local change in average duration $\left|\frac{\Delta \bar{D}_{\tau}}{\Delta \tau}\right|$ is a candidate measure of rate of change of an attractor of $f_{\tau}$, since it exists in all models $f$ and is coordinate-independent. It is ambiguous how large $\Delta \tau$ should be, however. Furthermore, the average duration $\bar{D}_{\tau}$ is only a proxy for the position of an attractor in phase space; an attractor can move even if $\frac{\Delta \bar{D}_{\tau}}{\Delta \tau}=0$. This explains the consistent deviation of single $\Delta \tau$
durations from the predicted and locally constant
$\bar{D}_{\tau}=82$ kyr in the inset of Fig. 3bi.

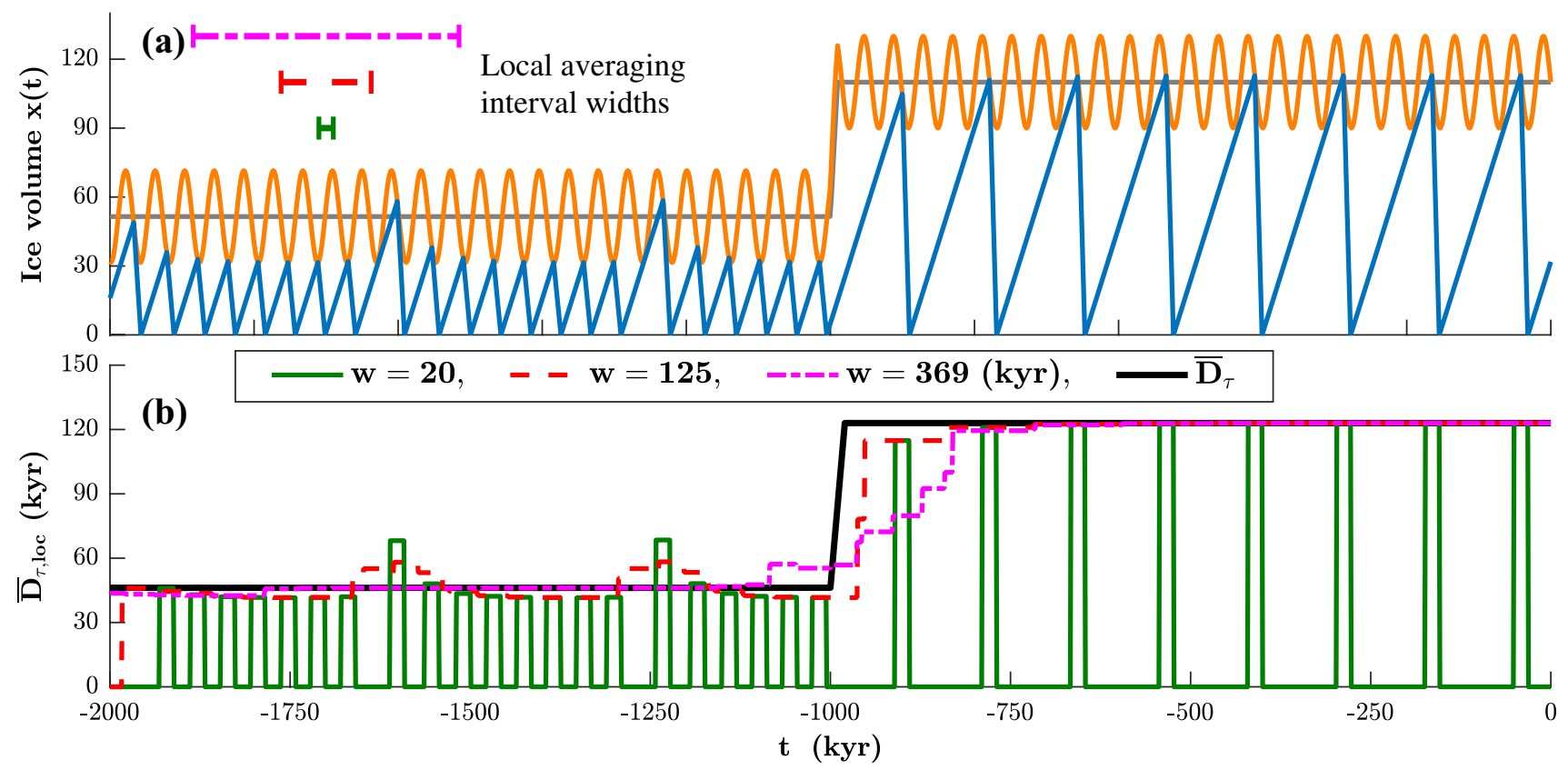

Fig. 6 Illustration of the difficulty estimating a local average frequency. a Ice volume from H07 (blue sawtooth), threshold of glacial termination (orange) and mean threshold of glacial termination $R(t)$ (grey). b Shows estimates of the local average duration $\bar{D}_{\tau, l o c}$ between glacial terminations in the $\mathrm{H} 07$ model with periodic forcing $\left(T_{f}=41\right.$ kyr), for different window widths. b $\bar{D}_{\tau, l o c}$ (green solid, red and magenta dashed) are running averages of durations in sliding win- dows of width $w=[20,125,369] \mathrm{kyr}$, or $0 \mathrm{kyr}$ if there are no durations in a window. The frozen time average duration $\bar{D}_{\tau}$ (black solid) is shown for reference. Prior to $-990 \mathrm{kyr}$ the steady state solution has a period of $9 \times 41=369 \mathrm{kyr}$, but an average duration $\bar{D}_{\tau}=46.125$ kyr. The mean threshold of glaciation $R(t)$ is ramped from 51.5 to 110 over $10 \mathrm{kyr}$ ( -990 to $-1000 \mathrm{kyr})$. Other parameters are $\mu=1, T_{\text {decay }}$ and $A=20$ 


\section{Is there a 100 kyr world?}

The late Pleistocene $(\sim-800-0 \mathrm{kyr})$ is sometimes referred to as the "100 kyr world", carrying the implicit notion that the Earth system has settled in a stationary mode with a dominant time scale of $100 \mathrm{kyr}$ (Fig. 1a). This view, originating from the closeness to the $100 \mathrm{kyr}$ component of eccentricity (an astronomical parameter), is supported by the rate of increase of mean ice volume seemingly levelling off (Clark et al. 2006; Mudelsee and Schulz 1997), and that the Fourier spectrum over the last $\sim 800 \mathrm{kyr}$ is centred around $100 \mathrm{kyr}$.

We propose on the contrary, following (Huybers 2007), that the glacial period increased gradually from $\sim 80 \mathrm{kyr}$ around $-1200 \mathrm{kyr}$ to $\sim 120 \mathrm{kyr}$ at present day. The change from $\sim 40$ to $\sim 80 \mathrm{kyr}$ long cycles at $-1200 \mathrm{kyr}$ can be a shift from $1 \times 41$ to $2 \times 41$ kyr obliquity frequency locking, and/or $2 \times 21$ to $4 \times 21 \mathrm{kyr}$ precession locking. We base this claim on durations between major glacial terminations and a wavelet spectrum of the LR04 stack (see Fig. 1b); both quantities increase rather rapidly around $-1200 \mathrm{kyr}$ and show a steady but irregular increase towards present time.

\subsection{Identifying the shift to longer periods}

While Huybers (2007) observed that the mean period of global ice volume variations increases over time, we make the stronger claim that an abrupt shift from 40 to $80 \mathrm{kyr}$ long durations occurred around $-1200 \mathrm{kyr}$. We base this claim on our identification of major glacial terminations, which unlike spectral decomposition ignores glacial cycle shape and is unaffected by time-frequency resolution.

A disadvantage of using glacial termination events is that it is unclear what constitutes a major termination, and whether it is meaningful to characterise glacial cycles by termination events. Nevertheless, we believe that our identification of major terminations is sufficiently robust to support the claim that the duration shifted abruptly from $\sim 40$ to $\sim 80 \mathrm{kyr}$ around $-1200 \mathrm{kyr}$.

\subsection{Testing for trend after the MPT}

It appears that the durations between successive glacial terminations are increasing over time starting at the onset of the MPT around $-1200 \mathrm{kyr}$.

We evaluate whether this trend is statistically significant, using a variation on the Mann-Kendall test (Kendall 1955; Mann 1945). Our null hypothesis H0 is that the sequence of thirteen durations from - $1126 \mathrm{kyr}$ until present is generated by a process with stationary mean, and that any observed monotonicity is by chance. Since $\tilde{D}_{i}=D_{i}-D_{\text {mean }}$, successive deviations from the mean duration $D_{\text {mean }}=91$ kyr are correlated, we immediately reject a white noise process as assumed in the standard Mann-Kendall test. Instead, we model them as an AR(1) process, such that $\tilde{D}_{i+1}=\alpha \tilde{D}_{i}+\sigma_{d} \xi_{i}$, where $\xi_{i}$ are independent Gaussian zero mean and unit variance elements. The parameters $\alpha=0.6$ and $\sigma_{d}=14.5 \mathrm{kyr}$ are the standard estimates of lag 1 and 0 autocorrelation coefficients, respectively.

We test the hypothesis using the Kendall $\tau$ test statistic for monotonicity $\tau_{K}$, based on the number of ordered and disordered pairs in a sequence. $\tau_{K}=1$ for a perfectly ordered sequence and $\tau_{K}=0$ for sequence with equally many ordered and disordered pairs (see "Appendix A" for a definition of $\left.\tau_{K}\right)$. We evaluate $\tau_{K}$ for $2 \cdot 10^{4}$ samples of the $\mathrm{AR}(1)$ process. As indicated in Fig. 7 , it is unlikely $(p<0.05)$ to observe the test statistic in durations from data, assuming that the durations follow an AR(1) process. Therefore, we reject the null hypothesis of no trend.

Adding age model uncertainty to the Monte Carlo sequences of durations only makes it more difficult to reject HO. Furthermore, slightly different choices of major glacial terminations, or the use of an untuned record, does not influence the conclusion of the test.

\subsection{Consequences for modelling the MPT}

Some explanations for the MPT do not reproduce the sequence of successively longer durations between glacial terminations in data as naturally as RFL. Instead, they produce long period cycles at the onset of the MPT which shorten towards the present as a parameter is ramped.

The Maasch and Salzman 1990 model in Fig. 8 is one such model (Maasch and Salzman 1990). The inconsistency with data is evident when comparing the model durations with those in the LR04 stack (Fig. 1). Another such model

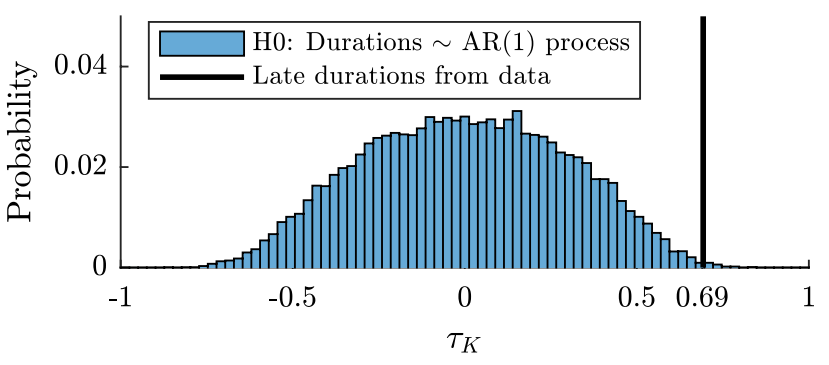

Fig. 7 Histogram shows a Monte Carlo distribution of the Kendall tau $\left(\tau_{K}\right)$ test statistic, under the null hypothesis $\mathrm{H} 0$ that the sequence of durations between glacial terminations from $-1126 \mathrm{kyr}$ follow an $\mathrm{AR}(1)$ process. Larger $\tau_{K}$ indicates a more monotonic sequence. Black line shows the test statistic $\tau_{K}$ for durations in an ice volume proxy (Fig. 1). $\tau_{K}$ under $\mathrm{H} 0$ exceeds the observed $\tau_{K}$ only in $5 \%$ of the cases. For details, see Sect. 6.2 


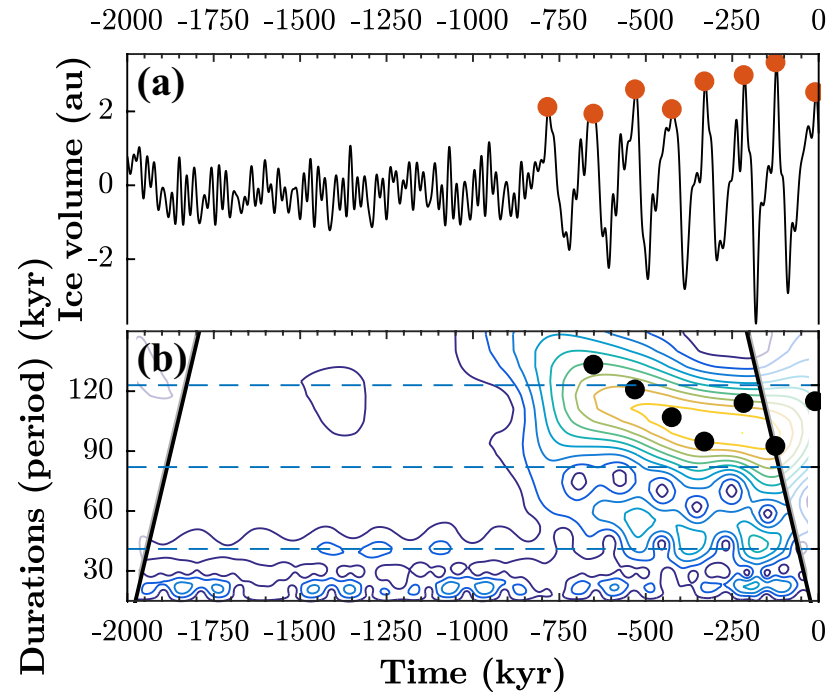

Fig. 8 Simulation of the Maasch and Salzman model in Maasch and Salzman (1990) forced by Summer solstice insolation at 65 degrees North. a Global ice volume over time (black), with glacial terminations (red dots) at peaks chosen for simplicity to be above 1.2 normalised ice volume units and spaced at least $60 \mathrm{kyr}$ apart. Self-sustained cycles emerge around $-800 \mathrm{kyr}$ and shorten towards the present. b Durations and wavelets as in Fig. 1, except that contours start at $10 \%$ of the maximum wavelet amplitude

is the Tziperman and Gildor 2003 model (Tziperman and Gildor 2003).

Although different dynamical mechanisms are at play in these models, they have in common that a long period limit unforced cycle emerges near a region of slow motion in phase space. As a parameter is varied, the limit cycle moves farther from this region, shortening the internal period.

RFL on the other hand naturally explains both a sudden shift from 40 to $80 \mathrm{kyr}$ cycles and a gradual increase towards longer cycles, since the system can respond both smoothly and abruptly to an increasing internal period, due to the Devil's staircase structure (e.g. Fig. 4). We interpret the progression of durations as evidence against models like Maasch and Salzman 1990 and Tziperman and Gildor 2003, and for mechanisms that naturally produce increasing glacial cycle length, such as RFL.

\subsection{Eccentricity and RFL}

According to one view, the "100"-kyr world constitutes the time when $\delta^{18} \mathrm{O}$ is strongly associated with eccentricity, an astronomical parameter that modulates the amplitude of precession at periods $\sim 100 \mathrm{kyr}$ and $\sim 400 \mathrm{kyr}$. Previous studies have found evidence both for Lisiecki (2010), Rial (1999) and Rial et al. (2013) and not for Huybers (2007) a significant relationship between eccentricity and $\delta^{18} \mathrm{O}$ over the past approximately $1000 \mathrm{kyr}$. We investigate whether such a relation is consistent with RFL.

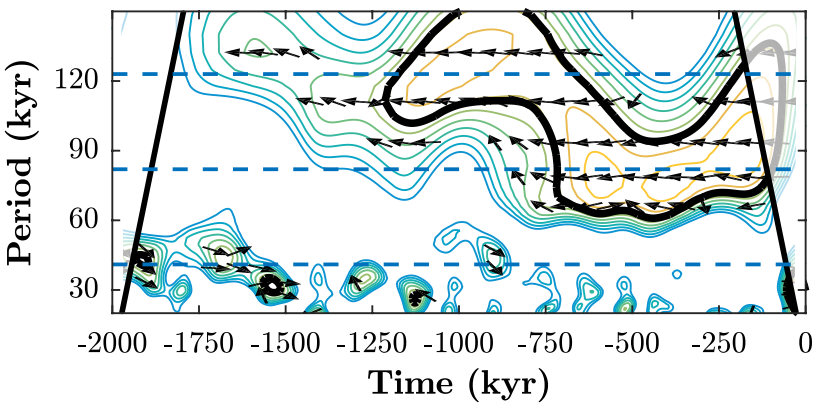

Fig. 9 Wavelet coherence between eccentricity and the LR04 stack (see "Appendix C"). Rightward arrows indicate that the signals are in-phase and leftward that they are anti-phase. Thin contour lines indicate a $7 \%$ increase in coherence, starting at 0.3 of the maximum coherence, which is 1 . The thick contour is the $95 \%$ confidence interval against red noise processes (see "Appendix C"). Outside the cone of influence (thick black side lines), edge effects are important

Wavelet coherency offers a way to assess phase relations between the LR04 stack and eccentricity without apriori choosing frequency bands to compare ("Appendix C"). It is a smoothed measure of local correlation in time-frequency space and is normalised by the local power of the time series (Grinsted et al. 2004; Torrence and Compo 1998). Thus, large cross-wavelet power does not result in large coherency only by virtue of sharing a time scale, which trivially results from two time series varying strongly on a $\sim 100 \mathrm{kyr}$ time scale (Maraun and Kurths 2004). The wavelet coherence shows a clear anti-phase association at a central period, which meanders between $\sim 80$ and $\sim 120 \mathrm{kyr}$ starting $\sim 1500$ to $\sim 1200 \mathrm{kyr}$ ago (Fig. 9). Note that the wavelet amplitude of the LR04 stack is weak at periods longer than $80 \mathrm{kyr}$ around time $-1000 \mathrm{kyr}$ (Fig. 1), so the coherence is not with the dominant mode of variability. The general picture is similar for the untuned H07 stack (Huybers 2007).

Is the anti-phase coherence between ice volume and eccentricity compatible with RFL? Judging from Fig. 9, the coherence around $-1200 \mathrm{kyr}$ ago is the most puzzling, since it is centred on period $\sim 120 \mathrm{kyr}$, while durations between glacial terminations are only $\sim 80 \mathrm{kyr}$ long. Therefore, the coherence must have another source, perhaps related to glacial cycle shape. Evaluating the cross-wavelet transform for two non-RFL models (Ashwin and Ditlevsen 2015; Maasch and Salzman 1990), and three RFL models (Paillard 1998; Paillard and Parrenin 2004; Feng and Bailer-Jones 2015) (the best-fit), we find that only the (Paillard 1998) model somewhat faithfully reproduces the phase coherence with eccentricity (not shown). The non-RFL models show negligible coherence with eccentricity after the MPT, which does not improve even if the forcing amplitude is increased relative to the published values. The RFL models (Paillard and Parrenin 2004; Feng and Bailer-Jones 2015) show some coherence, but only strongly after $-500 \mathrm{kyr}$. Although 
the model in Paillard (1998) is anti-phase coherent with eccentricity, it fails to reproduce the sequence of durations between glacial terminations across the MPT. It remains a challenge to formulate a model which both reproduces the sequence of durations and coherency with eccentricity. This should be possible for a model using RFL, but such a model must produce ice volume variability on the $\sim 120 \mathrm{kyr}$ time

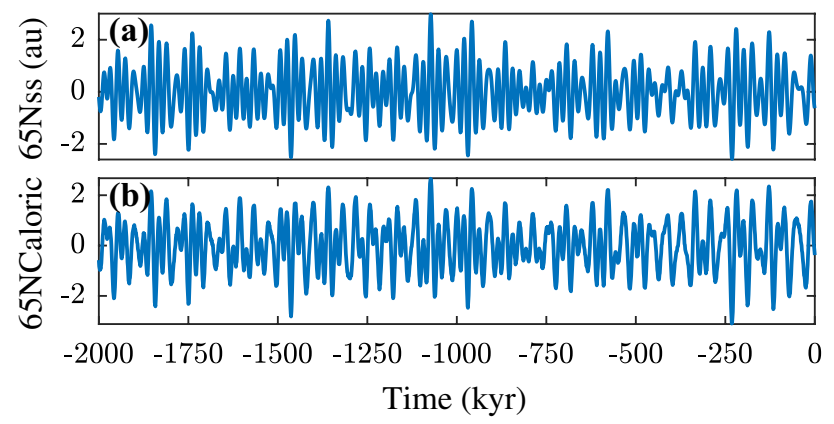

Fig. 10 Astronomical insolation curves. a Summer solstice insolation at 65 degrees North $(65 \mathrm{Nss})$, normalised to zero mean and unit variance (Laskar et al. 2004). The signal is approximately a linear combination of $33 \%$ normalised obliquity and $77 \%$ normalised precession, two modulated sinusoidal signals with central frequencies 41 and 22 kyr (Crucifix 2013). b Caloric summer insolation at 65N, consisting of roughly $50 \%$ obliquity and 50\% precession. Precession is amplitude modulated by eccentricity, a signal with dominant periods $\sim 100$ and $\sim 400 \mathrm{kyr}$ scale at the MPT, in addition to the variations with period $\sim 80 \mathrm{kyr}$ associated with glacial terminations.

\section{Non-harmonic forcing}

RFL is not restricted to harmonic forcing, but occurs also for astronomical, non-harmonic forcing. This is for instance the case for the Paillard and Parennin 2004 model (Fig. 11), forced by summer solstice insolation at 65 degrees North (65Nss, Fig. 10a). As a parameter is increased linearly, durations first cluster around $41 \mathrm{kyr}$, then shift abruptly to cluster around $82 \mathrm{kyr}$ at $-1000 \mathrm{kyr}$, after which they increase gradually until present. The shift to $80 \mathrm{kyr}$ durations is later than in proxy data (Fig. 1) and there are some short and long durations not clear in the proxy record, but overall the glacial terminations coincide well.

Multi-frequency forcing generally produces Devil's staircases with shorter steps of constant duration, making them look "smooth" (e.g. Fig. 12). This is apparently a problem for RFL since it relies on rapid jumps in durations. However, RFL can still be relevant as demonstrated by $\mathrm{H} 07$ forced by caloric summer insolation at $65 \mathrm{~N}$ consisting of an equal amount of obliquity and precession (Figs. 10b, 12, 13) (Tzedakis et al. 2017). The median and mode of the distribution of durations change more abruptly than the mean, which reflects that the gradual increase in average duration is caused by a gradual redistribution of durations between

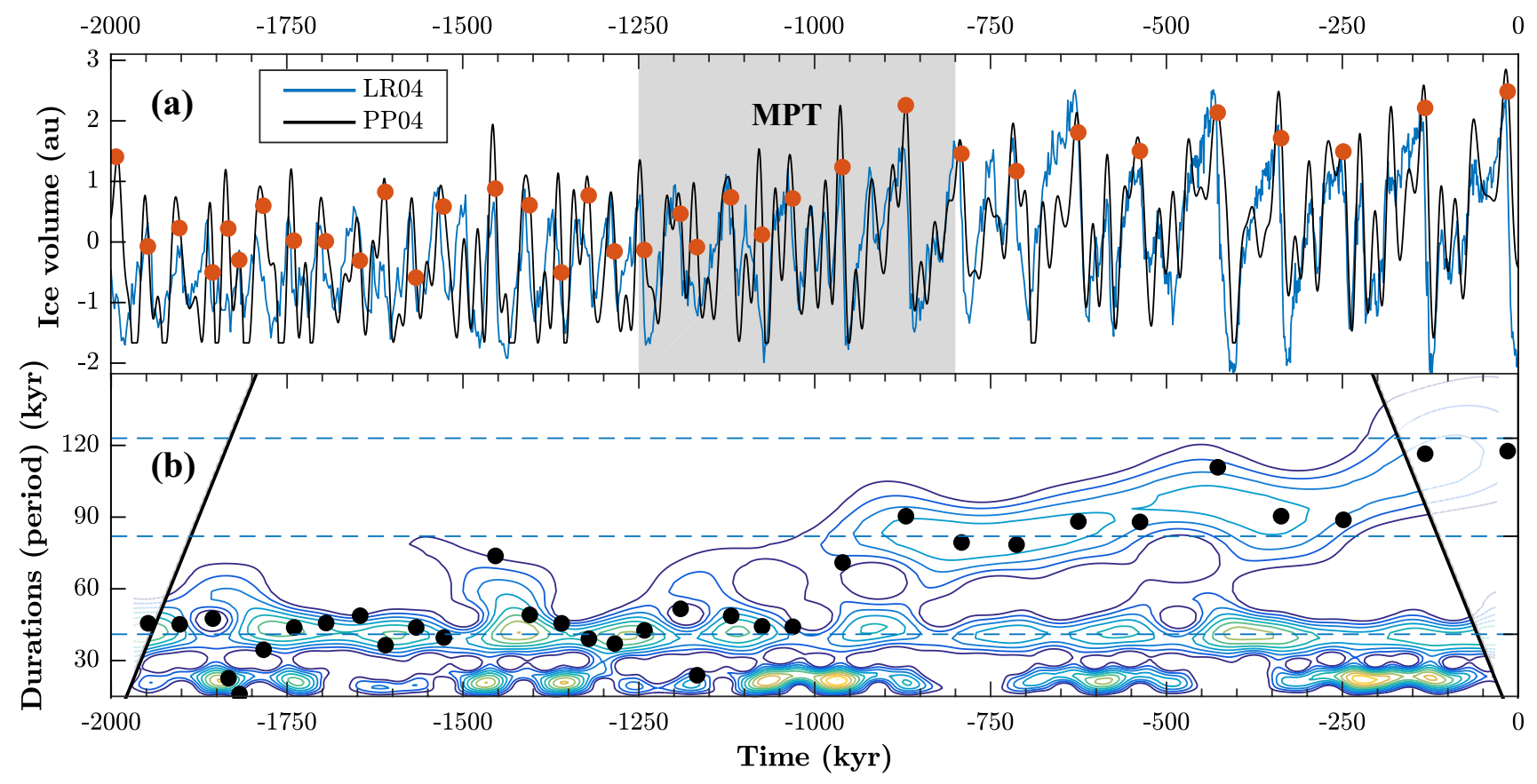

Fig. 11 Simulation of the Paillard and Parennin 2004 model in Paillard and Parrenin (2004) forced by Summer solstice insolation at 65 degrees North (Fig. 10a). a Model ice volume over time (black) contrasted with the LR04 stack (blue) (Fig. 1), with glacial terminations (red dots) at times when a switch in Southern ocean circulation occurs. b Durations and wavelets as in Fig. 1 


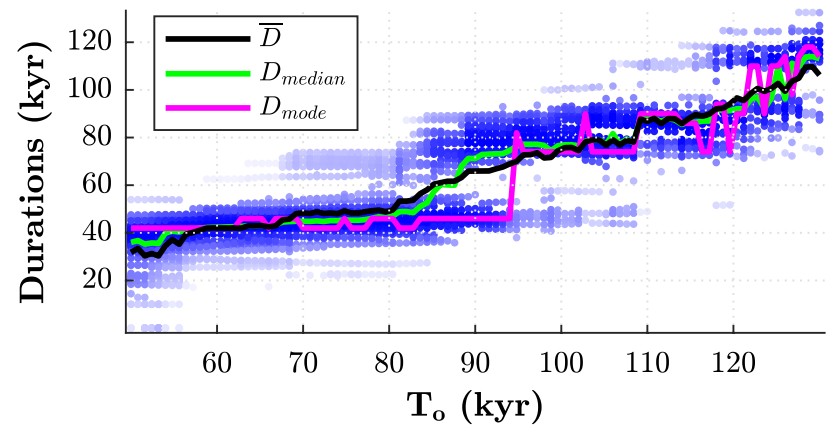

Fig. 12 Devil's staircases of H07 forced by caloric summer insolation (equal amounts of obliquity and precession, Fig. 10b). The average duration $\bar{D}$ (black line) as function of internal period $T_{o}$ is gradually increasing, whereas the median $D_{\text {median }}$ and the mode $D_{\text {mode }}$ are more step-like. Blue dots are the population of durations for fixed internal period; darker colours indicate higher density of durations. $D_{\text {mode }}$ is defined from binning the durations; $D_{\text {mode }}$ is the mean of the edges of the 4-kyr bin with the highest frequency. All quantities are evaluated from $-2000 \mathrm{kyr}$ to the present. Model parameters as in Fig. 13

clusters, rather than a gradual increase of the most typical durations. In a simulation with time-dependent ramping parameter, the local-in-time distribution of durations cannot be sampled well. Therefore the majority of the realised durations come from the dominant clusters of durations, which can give the impression that durations shift rapidly, in spite of the average duration changing gradually (Figs. 12, 13).
Multi-frequency forcing gives rise to many interesting phenomena regarding predictability of solutions, see for instance (Ashwin et al. 2018; Crucifix 2013; Grebogi et al. 1984; Imbrie and Imbrie 1980; Le Treut and Ghil 1983; Mitsui et al. 2015; de Saedeleer et al. 2013; Tziperman et al. 2006). Importantly, however, these phenomena are not essential to RFL. Whether solutions are truly frequency locked or depend on initial conditions is irrelevant, as long as durations undergo abrupt change and tend to cluster.

We conclude that RFL, clearly understood under periodic forcing, also is relevant for astronomical forcing. Indeed, recent studies provide evidence for the long-standing hypothesis that a combination of precession and obliquity paces the glacial cycles (Feng and Bailer-Jones 2015; Huybers 2011; Tzedakis et al. 2017). Differences between periodic and multi-frequency forcing exist, but are not crucial for modelling the MPT with RFL.

\section{Relevance for complex models and physical mechanisms}

We see two practical uses of our description of RFL: to guide modelling of the MPT in complex models, and to drive the search for slowly changing climate variables.

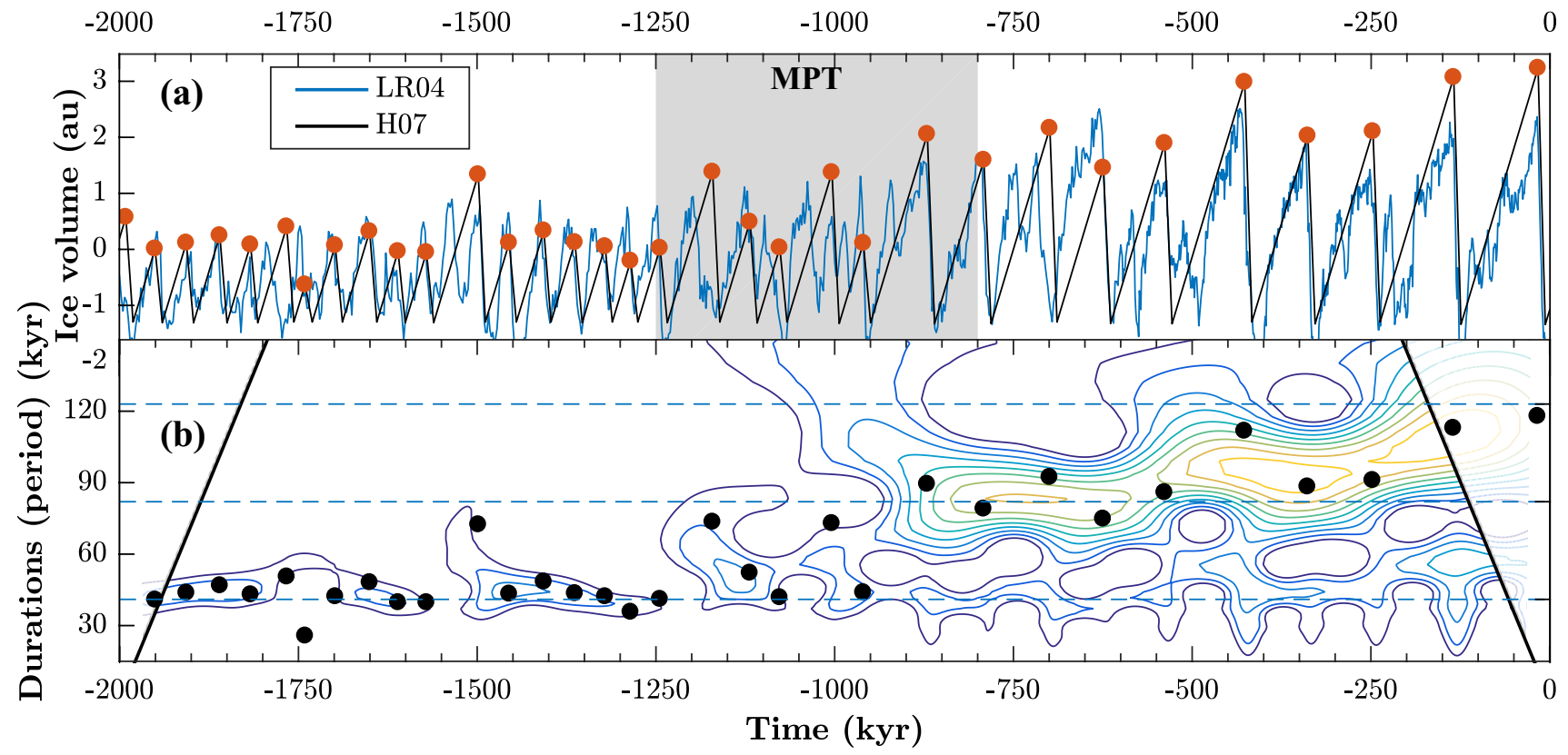

Fig. 13 Simulation of the H07 model forced by caloric summer insolation at $65 \mathrm{~N}$, a sum of equal amounts obliquity and precession (Fig. 10b). a Model ice volume (black) shown with the LR04 stack (blue) (Fig. 1), with glacial terminations (red dots) at times when a threshold of deglaciation is reached. b Durations and wavelets as in Fig. 1. The threshold of deglaciation increases linearly as $R(t)=40+0.04(t+2000)$, and forcing amplitude is $A=26$. All other parameters are as in Sect. 2 


\subsection{Relevance for complex models}

While climate physics are highly simplified in conceptual models like H07, their dynamics are well understood. The opposite holds true for earth system models (ESMs), which resolve multiple processes of climate in detail. To learn if the dynamical mechanism of RFL applies to such a model, we could in theory produce an Arnold tongue diagram as for H07 (Fig. 4). However, since running ESMs is computationally expensive this is presently not possible. Nevertheless, it might be possible to detect signatures of RFL from only few model runs.

First, one should investigate whether the glacial cycles are self-sustained by fixing model parameters at plausible values and fixing the insolation field at its mean value. This is the case if, after a transient time, variations in ice volume on the order of 10-100 kyr persist.

The next step is to sparsely sample an Arnold tongue diagram. First, a ramping parameter must be chosen. This does not have to be a scalar, but can be a function like a parametrisation, as long as its change over time is well defined. The parameter should be one that feasibly could influence the internal period of glacial cycles.

If changing the parameter changes the internal period, then one can compare the average duration of (insolation variation) forced and unforced solutions. If the average duration of the forced solutions is close to either 40 or $80 \mathrm{kyr}$ and remains close even under parameter perturbations that change the internal period, then this is an indication that the system is frequency locked to insolation in a way relevant for the glacial cycles. In that case, there is good reason to research RFL more closely in the model.

Ashkenazy (2006) suggested that synchronisation can be detected by running the system from multiple initial conditions and see if solutions converge. This procedure is not enough for us; we need to know if the internal period can be shifted appropriately with a change in parameter, and we need to know if the durations can robustly cluster on 40 and $80 \mathrm{kyr}$.

\subsection{Ramped climate variables}

To evaluate whether RFL caused the MPT one must identify slowly changing climate variables. Two such candidate variables are atmospheric $\mathrm{CO}_{2}$ and atmospheric or oceanic temperatures. Since less $\mathrm{CO}_{2}$ leads to a generally cooler atmosphere, it can be viewed as a proxy for global average atmospheric temperature. Local cooling can occur for other reasons, however.

There are currently no direct measurements of atmospheric $\mathrm{CO}_{2}$ across the MPT, but a recent reconstruction back to -2000 kyr suggests that the mean $\mathrm{CO}_{2}$ did not change in the mean until at least $-1300 \mathrm{kyr}$ (Hönisch et al. 2009).
Since the reconstruction implies that $\mathrm{CO}_{2}$ fell $31 \mathrm{ppm}$ by $-700 \mathrm{kyr}$, the decrease in $\mathrm{CO}_{2}$ must either have been rapid and driving the MPT, or a consequence of it. A rapid change in $\mathrm{CO}_{2}$ is still consistent with RFL, but in that case RFL does not explain the abrupt increase in cycle length at the MPT, and instead one must find an explanation for the rapid increase in $\mathrm{CO}_{2}$. However, the planned European BEOIC deep ice core drilling in Antarctica can hopefully improve estimates of $\mathrm{CO}_{2}$ across the MPT.

There is evidence of a gradual deep ocean cooling since the onset of northern Hemisphere glaciation 2.7 Myr ago (Lisiecki and Raymo 2005). How much of this cooling occurred across the MPT is not known, however. The reconstruction of deep water temperatures by Elderfield et al. (2012) indicates a gradual cooling in the mean from - $1300 \mathrm{kyr}$ until present, but also a puzzling warming from -1500 to -1300 kyr. Therefore, glacial cycle length does not appear to have a direct relation with mean deep ocean temperature. However, it may be that sea surface temperatures in the vicinity of major ice sheets are more relevant for glacial dynamics. If so, detailed and reliable reconstruction of such temperatures is necessary to evaluate whether they act as ramped climate variables in RFL.

Another slowly varying parameter could be the erosion of regolith. According to this hypothesis soft material under ice sheets eroded throughout the Pleistocene, enabling them to grow larger before collapsing Clark and Pollard (1998). The hypothesis is difficult to test empirically, however.

In addition to the candidate ramping climate variables mentioned, there may be others that are relevant for the MPT. RFL motivates the search for other such climate variables. These might not only be relevant for RFL, but for any mechanism of the MPT invoking deterministic bifurcation.

\section{Criteria for RFL}

Having demonstrated RFL in H07 and Paillard and Parennin 2004, we ask in which models RFL is most likely to be relevant.

We expect RFL in all models similar to H07, that is, models with a critical threshold of deglaciation (explicit or not), two intrinsic growth and decay states, additive forcing, and a climate variable that naturally controls the internal period.

Furthermore, RFL is facilitated by dynamics focussed on a single strongly attracting limit cycle. This is because solutions to the system $f$ with ramped parameters then track frozen solutions of $f_{\tau}$ well, and because it is difficult for perturbations to bring solutions away from the neighbourhood of the attractor.

Crucially, a model using RFL needs a parameter that can increase the internal period by $100 \mathrm{kyr}$. The models in e.g. (Le Treut and Ghil 1983 and Maasch and Salzman 1990) 
are therefore difficult to reconcile with RFL since the internal periods are on the order of 10 and 100 kyr respectively, and do not change much within the physical range of model parameters.

Lastly, we note that e.g. excitable systems and dissipative resonant oscillators (Crucifix 2012) also can undergo a rapid change in durations due to frequency locking related phenomena, although they are not self-sustained oscillators. Self-sustained oscillators are distinguished by having an internal period $T_{o}(R)$ through which we can define Arnold tongue diagrams and Devil's staircases; for non-selfsustained oscillators we have to define these through parameters $R$ directly. Furthermore, it has been argued that the term frequency locking should be restricted to self-sustained oscillators (Marchionne et al. 2018; Pikovsky et al. 2001), why it makes sense to define RFL for self-sustained oscillators only.

\section{Conclusions}

The glacial cycles did not enter a stationary $100 \mathrm{kyr}$ world at the MPT; instead, durations between glacial terminations shifted abruptly from $\sim 40$ to $\sim 80 \mathrm{kyr}$ around -1200 kyr, followed by a gradual increase (Fig. 1). The dynamical mechanism ramping with frequency locking (RFL) naturally explains this progression of durations. As the internal period of a model glacial cycle model increases gradually, frequency locking to insolation variations causes the durations between glacial terminations to increase sometimes abruptly and sometimes gradually.

The RFL mechanism is rather general and explains the behaviour of a range of models describing glacial cycles and the MPT (Ashkenazy 2006; Crucifix et al. 2011; Feng and Bailer-Jones 2015; Huybers 2007; Mitsui et al. 2015; Paillard 1998; Paillard and Parrenin 2004; Tzedakis et al. 2017).

Here we described how RFL can be understood in terms of a dynamical system $f$ and a frozen system $f_{\tau}$ with parameters $R(t)$ fixed at times $t=\tau$. The average duration $\bar{D}_{\tau}$ defined for $f_{\tau}$ provides some information about single durations in solutions $x(t)$ to $f$ around $t=\tau$, but since $\bar{D}_{\tau}$ is defined asymptotically, one must interpret solutions to $f$ in terms of $f_{\tau}$ with care.

Model behaviour can be understood from considering parameter paths through Arnold tongue diagrams and corresponding Devil's staircases (Figs. 3, 4, 5). These diagrams as functions of frozen time $\tau$ depend on

- the change in average duration $\bar{D}_{\tau}\left(T_{o}\right)$ as function of $T_{o}$,

- the change in internal period $T_{o}(R)$ as function of $R$,

- the change in parameters $R(\tau)$ as function of time $\tau$, and

- the amplitude $A$ of the forcing.
This decomposition clarifies different ways in which the average duration can change abruptly in models of the class $f$. For instance, the abruptness of the change in $\bar{D}_{\tau}$ can be adjusted either by changing the forcing amplitude $A$ or the ramping of $R(t)$. While the effects of changing $A$ or the ramping of $R(t)$ are typically easy to guess, we are not aware of any general rules dictating the widths of particular Arnold tongues. Such understanding may be researched further.

RFL is relevant also for multi-frequency astronomical forcing. Multi-frequency forcing tends to make Devil's staircases less abrupt, but durations can still increase rapidly when a model parameter is slowly ramped.

It remains a challenge to reconcile the phase coherence between eccentricity and ice volume proxies at the MPT with the $\sim 80 \mathrm{kyr}$ durations between glacial terminations. The RFL model Paillard 1998 reproduces well the phase coherence, but not the durations. Two RFL models and two non-RFL models with a strong self-sustained limit cycles forced by precessionheavy forcing (modulated by eccentricity) fail to reproduce the phase coherency.

The RFL mechanism provides an explanation for the MPT without the climate system entering a new mode of operation. A shift from $\sim 40 \mathrm{kyr}$ long to $\sim 80 \mathrm{kyr}$ long cycles due to frequency locking to obliquity and precession, is consistent with data (Fig. 1), and is used in models (Huybers 2007; Paillard and Parrenin 2004). This warrants further study of frequency locking characteristics of models throughout the model hierarchy, as well as a search for gradually increasing climate parameters. Some models use a rapidly ramped parameter to accelerate the increase in durations between glacial terminations at the MPT, but such a ramping begs for justification that a model relying solely on frequency locking does not require.

Acknowledgements We thank Peter Ashwin for valuable discussions and three anonymous reviewers for constructive comments. Insolation codes were adapted from the palinsol R package by M. Crucifix. Matlab code for evaluating wavelet coherency was provided by A. Grinsted. This research has been funded by the European Union's Horizon 2020 innovation and research programme for the ITN CRITICS under the Marie Skłodowska-Curie Grant agreement no. 643073.

Open Access This article is distributed under the terms of the Creative Commons Attribution 4.0 International License (http://creativeco mmons.org/licenses/by/4.0/), which permits unrestricted use, distribution, and reproduction in any medium, provided you give appropriate credit to the original author(s) and the source, provide a link to the Creative Commons license, and indicate if changes were made.

\section{Appendix A: Kendall's tau}

Kendall's tau, here denoted $\tau_{K}$, when testing for monotonicity of a sequence $\left\{D_{i}\right\}_{i=1}^{n}$ is defined as:

$$
\tau_{K}=\frac{n_{c}-n_{d}}{\sqrt{n_{0}^{2}-n_{0} n_{1}}},
$$


where $n_{c}-n_{d}=\sum_{i<j} \operatorname{sign}\left(D_{j}-D_{i}\right)$ is the number of pairs $\left(D_{i}, D_{j}\right)$ that are ordered $\left(D_{j}>D_{i}\right)$ minus the number that is disordered, $n_{0}=n(n-1) / 2$ is the total number of pairs and $n_{1}=\sum_{k} t_{k}\left(t_{k}-1\right) / 2$ is the sum of the number of tied elements $t_{k}$ in the $k$ :th group of tied elements. For example, the sequence $\{1,2,2\}$ has two ordered pairs $(1,2)$ and $(1,2)$, zero disordered pairs, and one tied pair $(2,2)$. Hence, $k=1$ such that $n_{c}-n_{d}=2, n_{0}=3$ and $n_{1}=1$, giving $\tau_{K}=2 / \sqrt{6} \approx 0.82$.

\section{Appendix B: Wavelets}

Wavelet spectra are estimated with the MATLAB function cwt, using Morlet basis functions with bandwidth parameter $\omega_{0}=6$ (Torrence and Compo 1998), softwaree by. Contours show wavelet amplitude (square root of variance) relative to the maximum, incremented in evenly spaced percentage units. The cone of influence marks the $e$-folding time of the amplitude of a discontinuity at the edge of the time interval. Inside the cone of influence edge effects are negligible (Torrence and Compo 1998).

\section{Appendix C: Cross-wavelet transform and wavelet coherency}

The cross-wavelet transform of two time series $X$ and $Y$ is given by $W_{n}^{X Y}(s)=W_{n}^{X}(s) W_{n}^{Y *}(s)$, where $W_{n}^{X}(s)$ is the wavelet transform of $X$ at discrete time $n$ and scale $s$, and $W_{n}^{Y *}(s)$ is the complex conjugate of the wavelet transform of $Y$. The wavelet squared coherency is defined as:

$R_{n}^{2}(s)=\frac{\left|S\left(s^{-1} W_{n}^{X Y}(s)\right)\right|^{2}}{\left|S\left(s^{-1} W_{n}^{X}(s)\right)\right|^{2}\left|S\left(s^{-1} W_{n}^{Y}(s)\right)\right|^{2}}$,

where $S$ is a smoothing function in space and time, found in Grinsted et al. (2004) and Torrence and Webster (1999), and $s$ is scale (approximately equal to period).

Significance levels for wavelet coherency between $X$ and $Y$ was estimated Monte Carlo by estimating the wavelet coherency between a large number (1000) of red noise processes with parameters fitted from $X$ and $Y$. The confidence levels are reliable if at least one of $X$ and $Y$ can be modelled as a red noise process (Maraun and Kurths 2004), which holds decently for the LR04 stack (Fig. 9). (Maraun and Kurths (2004) considered a white noise process, but we expect the conclusion to hold also for a red noise process). Large coherent regions in timeperiod space are more likely non-spurious than small ones (Maraun and Kurths 2004).

\section{Appendix D: Glacial terminations in LR04}

Major glacial terminations in the LR04 stack (Fig. 1) are identified at times $t=-[1948,1900,1863,1795,1748$, $1708,1655,1575,1535,1496,1456,1412,1372,1336$, 1290, 1248, 1198, 1126, 1038, 964, 876, 794, 718, 630, $536,434,341,252,140,18]$ kyr. We assume conservatively an age model uncertainty with constant standard deviation $6 \mathrm{kyr}$ over the past $2000 \mathrm{kyr}$ (Lisiecki and Raymo 2005), which gives a standard deviation $\sqrt{2} \cdot 6 \mathrm{kyr}$ on the durations between terminations, assuming somewhat wrongly that errors are independent and normally distributed.

\section{References}

Ashkenazy Y (2006) The role of phase locking in a simple model for glacial dynamics. Clim Dyn 27:421-431. https://doi.org/10.1007/ s00382-006-0145-5

Ashkenazy Y, Tziperman E (2004) Are the 41 kyr glacial oscillations a linear response to Milankovich forcing? Quat Sci Rev 23:18791890. https://doi.org/10.1016/j.quascirev.2004.04.008

Ashwin P, Ditlevsen P (2015) The middle Pleistocene transition as a generic bifurcation on a slow manifold. Clim Dyn. https://doi. org/10.1007/s00382-015-2501-9

Ashwin P, Camp CD, von der Heydt AS (2018) Chaotic and non-chaotic response to quasiperiodic forcing: limits to predictability of ice ages paced by Milankovitch forcing. Dyn Stat Clim Syst. https ://doi.org/10.1093/climsys/dzy002

Baer S, Ernaux T, Rinzel J (1989) The slow passage through a hopf bifurcation: delay, memory effects, and resonance. SIAM J Appl Math 49(1):55-71. https://doi.org/10.1137/0149003

Berger AL (1978) Long-term variations of daily insolation and quaternary climatic changes. J Atmos Sci. https://doi.org/10.1175/15200469(1978)035<2362:LTVODI>2.0.CO;2

Cartwright M, Littlewood J (1945) On non-linear differential equations of the second order. J Lond Math Soc 1-20(3):180-189. https:// doi.org/10.1112/jlms/s1-20.3.180

Clark PU, Pollard D (1998) Origin of the middle Pleistocene transition by ice sheet erosion of regolith. Paleoceanography 13(1):1-9. https://doi.org/10.1029/97PA02660

Clark PU, Archer D, Pollard D, Blum JD, Rial JA, Brovkin V, Mix AC, Pisias NG, Roy M (2006) The middle Pleistocene transition: characteristics, mechanisms, and implications for the long-term changes in atmospheric $\mathrm{pCO}_{2}$. Quat Sci Rev 25:3150-3184. https ://doi.org/10.1016/j.quascirev.2006.07.008

Crucifix M (2012) Oscillators and relaxation phenomena in Pleistocene climate theory. Philos Trans R Soc Lond A 370(1962):1140-1165. https://doi.org/10.1098/rsta.2011.0315

Crucifix M (2013) Why could ice ages be unpredictable? Clim Past 9:2253-2267. https://doi.org/10.5194/cp-9-2253-2013

Crucifix M, Lenoir G, de Saedeleer B (2011) The mid-Pleistocene transition and slow fast dynamics. In: EGU2011-3629-1, EGU general assembly 2011, geophysical research abstracts, vol 13, poster

Daruka I, Ditlevsen PD (2015) A conceptual model for glacial cycles and the middle Pleistocene transition. Clim Dyn 46:29-40. https ://doi.org/10.1007/s00382-015-2564-7

Ditlevsen PD (2009) Bifurcation structure and noise-assisted transitions in the Pleistocene glacial cycles. Paleoceanography. https:// doi.org/10.1029/2008PA001673 
Do Y, Lopez JM (2012) Slow passage through multiple bifurcation points. Am Inst Math Sci 18(1):95-107. https://doi.org/10.3934/ dcdsb.2013.18.95

Elderfield H, Ferretti P, Greaves M, Crowhurst S, McCave IN, Hodell D, Piotrowski AM (2012) Evolution of ocean temperature and ice volume through the mid-Pleistocene climate transition. Science 337(6095):704-709. https://doi.org/10.1126/science.1221294

EPICA Community Members (2004) Eight glacial cycles from an Antarctic ice core. Nature 429:623-628. https://doi.org/10.1038/ nature 02599

Feng F, Bailer-Jones CAL (2015) Obliquity and precession as pacemakers of Pleistocene deglaciations. Quat Sci Rev 122:166179. https://doi.org/10.1016/j.quascirev.2015.05.006

Gildor H, Tziperman E (2000) Sea ice as the glacial cycles' climate switch: role of seasonal and orbital forcing. Paleoceanography 15(6):605-615. https://doi.org/10.1029/1999PA000461

Glass L, Mackey MC (1979) A simple model for phase locking of biological oscillators. J Math Biol 7:339-352. https://doi. org/10.1007/BF00275153

Grebogi C, Ott E, Pelican S, Yorke JA (1984) Strange attractors that are not chaotic. Phys D 13:261-268. https://doi. org/10.1016/0167-2789(84)90282-3

Grinsted A, Moore JC, Jevrejeva S (2004) Application of the cross wavelet transform and wavelet coherence to geophysical time series. Nonlinear Process Geophys 11(5/6):561-566. https://doi. org/10.5194/npg-11-561-2004

Guckenheimer J, Hoffman K, Weckesser W (2003) The forced van der pol equation I: the slow flow and its bifurcations. SIAM J Appl Dyn Syst 2(1):1-35. https://doi.org/10.1137/S111111110 2404738

Huybers P (2006) Early Pleistocene glacial cycles and the integrated summer insolation forcing. Science 313:508-510. https://doi. org/10.1126/science. 1125249

Huybers P (2007) Glacial variability over the last two million years: an extended depth-derived agemodel, continuous obliquity pacing, and the Pleistocene progression. Quat Sci Rev 26:37-55. https:// doi.org/10.1016/j.quascirev.2006.07.013

Huybers P (2009) Pleistocene glacial variability as a chaotic response to obliquity forcing. Clim Past 5:481-488. https://doi.org/10.5194/ cp-5-481-2009

Huybers P (2011) Combined obliquity and precession pacing of late Pleistocene deglaciation. Nature 480:229-231. https://doi. org/10.1038/nature10626

Huybers P, Langmuir CH (2017) Delayed CO_2 emissions from midocean ridge volcanism as a possible cause of late-Pleistocene glacial cycles. Earth Planet Sci Lett 457:238-249. https://doi. org/10.1016/j.eps1.2016.09.0

Hönisch B, Hemming G, Archer D, Siddall M, McManus JF (2009) Atmospheric carbon dioxide concentration across the mid-Pleistocene transition. Science 324(5934):1551-1554. https://doi. org/10.1126/science. 1171477

Imbrie J, Imbrie JZ (1980) Modeling the climatic response to orbital variations. Science 207:943-953. https://doi.org/10.1126/scien ce.207.4434.943

Imbrie J, Imbrie KP (1979) Ice ages: solving the mystery, 1st edn. MacMillan, London

Imbrie JZ, Imbrie-Moore A, Lisiecki L (2011) A phase-space model for Pleistocene ice volume. Earth Planet Sci Lett 307:94-102. https:// doi.org/10.1016/j.eps1.2011.04.018

Kendall M (1955) Rank correlation methods, 2nd edn. Hafner Publishing Co., Oxford

Laskar J, Robutel P, Joutel F, Gastineau M, Correia ACM, Levrard B (2004) A long-term numerical solution for the insolation quantities of the earth. Astron Astrophys 428:261-285. https://doi. org/10.1051/0004-6361:20041335
Le Treut H, Ghil M (1983) Orbital forcing, climatic interactions, and glaciation cycles. J Geophys Res 88(C9):5167-5190. https://doi. org/10.1029/JC088iC09p05167

Levi M (1990) A period-adding phenomenon. SIAM J Appl Math 50(4):943-955. https://doi.org/10.1137/0150058

Lisiecki LE (2010) Links between eccentricity forcing and the 100,000-year glacial cycle. Nature Geosci 3:349-352. https:// doi.org/10.1038/ngeo828

Lisiecki LE, Raymo ME (2005) A Plioene-Pleistocene stack of 57 globally distributed benthic $\delta^{18} O$ records. Paleoceanography 20:437-440. https://doi.org/10.1029/2004PA001071

Maasch KA, Salzman B (1990) A low-order dynamical model of global climatic variability over the full Pleistocene. J Geophys Res 95(D2):1955-1963. https://doi.org/10.1029/JD095iD02p01955

Mann HB (1945) Nonparametric tests against trend. Econometrica 13(3):245-259. https://doi.org/10.2307/1907187

Maraun D, Kurths J (2004) Cross wavelet analysis: significance testing and pitfalls. Nonlinear Process Geophys 11(4):505-514. https:// doi.org/10.5194/npg-11-505-2004

Marchionne A, Ditlevsen P, Wieczorek S (2018) Is the astronomical forcing a reliable and unique pacemaker for climate? A conceptual study. Phys D 380-381:8-16. https://doi.org/10.1016/j.physd .2018.05.004

Mitsui T, Crucifix M, Aihara K (2015) Bifurcations and strange nonchaotic attractors in a phase oscillator model of glacial-interglacial cycles. Phys D 306:25-33. https://doi.org/10.1016/j.physd .2015.05.007

Mudelsee M, Schulz M (1997) The mid-Pleistocene climate transtion: onset of 100 ka cycles lags ice volume build-up by $280 \mathrm{ka}$. Earth Planet Sci Lett 151:117-123. https://doi.org/10.1016/S0012 -821X(97)00114-3

Omta AW, Kooi BW, van Voorn GAK, Rickaby REM, Follows MJ (2015) Inherent characteristics of sawtooth cycles can explain different glacial periodicities. Clim Dyn 46:557-569. https://doi. org/10.1007/s00382-015-2598-x

Paillard D (1998) The timing of Pleistocene glaciations from a simple multiple-state climate model. Nature 391:378-381. https://doi. org/10.1038/34891

Paillard D, Parrenin F (2004) The Antarctic ice sheet and the triggering of deglaciations. Earth Planet Sci Lett 227:263-271. https://doi. org/10.1016/j.epsl.2004.08.023

Parrenin F, Paillard D (2003) Amplitude and phase of glacial cycles from a conceptual model. Earth Planet Sci Lett 214(1):243-250. https://doi.org/10.1016/S0012-821X(03)00363-7

Parrenin F, Paillard D (2012) Terminations vi and viii (530 and 720 kyr bp) tell us the importance of obliquity and precession in the triggering of deglaciations. Clim Past 8(6):2031-2037. https://doi. org/10.5194/cp-8-2031-2012

Pikovsky A, Rosenblum M, Kurths J (2001) Synchronization: a universal phenomenon in the nonlinear sciences, 1st edn. Cambridge University Press, Cambridge

van der Pol B, van der Mark J (1927) Frequency demultiplication. Nature 120:363-364. https://doi.org/10.1038/120363a0

Quinn C, Sieber J, von der Heydt AS, Lenton TM (2018) The midPleistocene transition induced by delayed feedback and bistability. Dyn Stat Clim Syst 3(1):1-17. https://doi.org/10.1093/clims ys/dzy005

Rial JA (1999) Pacemaking the ice ages by frequency modulation of earth's orbital eccentricity. Science 285(5427):564-568. https:// doi.org/10.1126/science.285.5427.564

Rial JA, Oh J, Reischmann E (2013) Synchronization of the climate system to eccentricity forcing and the 100,000-year problem. Nat Geosci 6:289-293. https://doi.org/10.1038/ngeo1756

de Saedeleer B, Crucifix M, Wieczorek S (2013) Is the astronomical forcing a reliable and unique pacemaker for climate? A conceptual 
study. Clim Dyn 40:273-294. https://doi.org/10.1007/s0038 2-012-1316-1

Salzman B, Verbitsky MY (1993) Multiple instabilities and modes of glacial rhythmicity in the Plio-Pleistocene: a general theory of late Cenozoic climatic change. Clim Dyn 9:1-15. https://doi. org/10.1007/BF00208010

Torrence C, Compo GP (1998) A practical guide to wavelet analysis. Bull Am Meteorol Soc 79(1):61-78. https://doi. org/10.2307/1907187

Torrence C, Webster PJ (1999) Interdecadal changes in the ensomonsoon system. J Clim 12(8):2679-2690. https://doi. org/10.1175/1520-0442(1999)012<2679:ICITEM>2.0.CO;2

Tzedakis PC, Crucifix M, Mitsui T, Wolff EW (2017) A simple rule to determine which insolation cycles lead to interglacials. Nature 542:427-432. https://doi.org/10.1038/nature21364
Tziperman E, Gildor H (2003) On the mid-Pleistocene transition to $100-k y r$ glacial cycles and the asymmetry between glaciation and deglaciation times. Paleoceanography 18(1):1-8. https://doi. org/10.1029/2001PA000627

Tziperman E, Raymo M, Huybers P, Wunsch C (2006) Consequences of pacing the Pleistocene $100 \mathrm{kyr}$ ice ages by nonlinear phase locking to milankovitch forcing. Paleoceanography $21: 1-11$. https ://doi.org/10.1029/2005PA001241

Publisher's Note Springer Nature remains neutral with regard to jurisdictional claims in published maps and institutional affiliations. 